\title{
EPILEPSIA E ORDEM DE NASCIMENTO
}

\author{
Paulo Cesar Sandler * \\ Ester Hadassa SANDLER **
}

\begin{abstract}
O corpo de doutrina ao qual se convencionou chamar Psiquiatria Social e que, em certos lugares tem a conotação de verdadeiro movimento 21, 28, tenta elucidar a influência de fatores ambientais no complexo quadro etiopatogênico das doenças mentais. Embora seu arcabouço teórico ainda esteja por ser montado $2,8,20,21,26,27$, existem métodos científicos válidos que têm sido aplicados na investigação em Psiquiatria Social. O método mais bem firmado é, sem dúvida, o epidemiológico ${ }^{16,19}$ que, mesmo não pretendendo fornecer respostas finais ou redes multifatoriais de causalidade ${ }^{19}, 20$, pode emprestar validade científica a facetas dos quadros etiológicos. A própria Organização Mundial de Saúde considera como valiosa a pesquisa orientada estatisticamente, tendo feito várias recomendações neste sentido ${ }^{14}$.
\end{abstract}

Dentro das variáveis sociais que a Psiquiatria Social enfoca, têm merecido especial atenção as variáveis micro-sociais ${ }^{20,21}$. Dentro destas, a quantificação de dados familiares objetivos tem ocupado grande parte da literatura, em relação a certas sindromes. $O$ dado mais bem estudado tem sido a ordem de nascimento dos pacientes, ou seja, a sua posição dentro da prole, e a sua possível ocorrência concomitante à prevalência ${ }^{11}$ das várias patologias psiquiátricas. A patologia mais pesquisada, em termos de número de trabalhos publicados, é sem dúvida a esquizofrenia ${ }^{4}, 10,24,25$, seguida do alcoolismo '.

A ordem de nascimento do paciente oferece larga gama de hipóteses subsequentes de trabalho, após ter sido estudada do ponto de vista estatístico: hipóteses que sejam testadas também pelo método estatístico, onde se controlem outras variáveis, concomitantemente (privação parental ${ }^{9}$, classes sociais ${ }^{17}$ e outras) e hipóteses que expliquem a natureza das associações estatísticas porventura encontradas. Estas hipóteses explicativas são necessariamente provenientes de outros modelos teóricos que não a própria epidemiologia, conforme a entidade nosológica que se enfoque, caracterizando um

Trabalho realizado mediante bolsa da Fundação de Amparo à Pesquisa do Estado de São Paulo, proc. 70/1183 e 72/299 do Departamento de Medicina Preventiva da Faculdade de Medicina da Universidade de São Paulo: * Residente de Psiquiatria da Faculdade de Medicina da Universidade de São Paulo, médico-assistente no Instituto Aché (Sāo Paulo); ** Estudante do 6." ano (Experimental) da Faculdade de Medicina da Universidade de São Paulo.

Nota dos autores - Deixamos expresso nosso agradecimento ao Professor Guilherme Rodrigues da Silva, chefe do Departamento de Medicina Preventiva da Faculdade de Medicina da Universidade de São Paulo, pelo apoio e incentivo. 
indispensável estudo multidisciplinar das doenças mentais 2, 16, 19, 27. Assim, por exemplo, em patologias ditas funcionais, como as neuroses e alguns quadros psicóticos, uma frequência maior ou menor de primeiros filhos no grupo de pacientes poderá ser explicada por modelos psicodinâmicos ou interacionais ${ }^{3}$. Em quadros patológicos cuja etiopatogenia é reconhecidamente orgânica, os modelos teóricos explicativos também o serão; há quadros onde uma interpretação verdadeiramente ambiental pode ser cabível, sendo admissiveis, em outros, explicações genéticas ${ }^{13}$.

Sem entrar no terreno destas discussões teóricas, o presente estudo cuida de uma patologia de interesse neuro-psiquiátrico óbvio e de crescente preocupação médico-social: a epilepsia.

\section{METODOS}

Foi escolhido o método de Greenwod-Yule ${ }^{11}$ e o teste de aderência, sendo $0 x^{2}$ modificado por Max Halperin ", ":, que é o mais preciso para testar efeitos de ordem de nascimento que se dispõe atualmente. No método de Greenwood-Yule monta-se um grupo controle que é comparado com o grupo de doentes: distribuem-se as frequências obtidas na casuistica em uma tabela de ordens de nascimento, conforme o tamanho da prole, ou seja, o número de filhos.

Este grupo controle de distribuiçōes hipotéticas decorre da hipótese nula do método: não existe associação entre a ordem de nascimento considerada dos pacientes (sua posicão ordinal dentro da prole de origem) e a prevalência da patologia em questão. Assim, o doente tem igual chance de nascer em qualquer ordem de nascimento. Em outras palavras, dada uma prole de $\mathbf{N}$ individuos, para uma ordem de nascimento $K$, a probabilidade de termos individuos afetados será dada por $K / N$.

\section{MATERIAL}

Foram usados dados hospitalares, com diagnósticos de certeza, em dois hospitais psiquiátricos da cidade de São Paulo ${ }^{\circ}$ Assim, a população de epilépticos que aparece neste estudo é composta de doentes que apresentam. todos eles, manifestaçoes psiquiátricas que justificaram a sua internação em hospital deste tipo. Incluem-se então as psicoses epilépticas, os transtornos de personalidade devidos à epilepsia, e outros transtornos não-psicóticos ligados à epilepsia. Um dos hospitais é uma clínica universitária, com todos os recursos diagnósticos, inclusive EEG de rotina, e que alberga doentes das classes econômicas menos favorecidas. $O$ outro é um hospital particular, que mantém convênio com entidade previdenciária federal, albergando doentes das classes econômicas média e superior: é um hospital particular antigo, dirigido por dois clinic'os experientes, que cuidam pessoalmente dos diagnósticos. Esta escolha foi feita em uma tentativa de ser controlada, de maneira qualitativa, a variável classe social, que modifica a prevalência das doenças mentais ${ }^{\circ}$. Foram rejeitados os casos nos quais o diagnóstico era duvidoso; a orientação diagnóstica nos dois hospitais é a mesma. Os irmãos mortos na primeira infância não foram considerados. Não foram excluidos os gêmeos, que foram tratados segundo MacMahon e col. 11. Para não trabalharmos com proles incompletas ${ }^{11}$, ${ }^{15}$, só foram considerados os pacientes que contavam mais de 15 anos de idade. Foi fixado "a priorl" o nivel de significância em 0.01 para aceitação dos resultados.

* Os hospitais utilizados foram a Clinica Psiquiátrica do Hospital das Clinicas da Faculdade de Medicina da Universidade de São Paulo e o Instituto Aché. Somos gratos aos diretores clinicos destes dois hospitais - Drs. H. M. Carvalho (Hospital das Clínicas) e Drs. Mário Yahn e Waldemar Cardoso (Instítuto Aché) - que nossibilitaram a อxecilcãn dก trahalhn 


\section{RESULTADOS}

Foram obtidos 238 propósitos para a análise estatística e a idade média da casuistica foi de 31,58 anos, o que permite afirmar que é muito pequena a probabilidade de estarmos trabalhando com proles incompletas (Tabela 1). A tabela 2 mostra a distribuição observada das frequências das ordens de nascimento e a tabela 3, a distribuição esperada, conforme o método de Greenwod-Yule. Mediante o método de Halperin ", com simplificação de Mantel e Halperin ":, obtém-se um x: de 46.16, quando se comparam as frequências observadas e esperadas (Tabela 4). Este $x^{*}$ é altamente significante. A título de comparação, aplicou-se o $x^{2}$ clássicco. sem modificacão, e sua inadequação torna-se evidente: sabe-se que a ordem de nascimento não segue a curva de distribuição do $\mathrm{x}^{2}$, e vários autores propuseram outros testes de aderência por esta razão ${ }^{5}$, .

\begin{tabular}{cc|cr}
\hline $\begin{array}{l}\text { Intervalo } \\
\text { de idade }\end{array}$ & Frequência & $\begin{array}{l}\text { Intervalo } \\
\text { de idade }\end{array}$ & Frequência \\
\hline $15-19$ & 95 & $35-39$ & 19 \\
$20-24$ & 44 & $40-44$ & 11 \\
$25-29$ & 29 & $45-49$ & 4 \\
$30-34$ & 20 & $50-54$ & 5 \\
\hline
\end{tabular}

Idade média: 31,58 anos

Tabela 1 - Frequencias de intervalo de idades observadas em 238 epilépticos. Cálculo la illade média desta casuistica.

Examinando-se a tabela 4, nota-se que a associação estatisticamente significante entre epilepsia e ordem de nascimento se dá na direcão dos primeiros e dos últimos nascidos.

Assim, de maneira sintética, em um grupo de 238 epilépticos brasileiros, internados em hospitais psiquiátricos, foi encontrada uma associaçāo estatistic amente significante entre a doenca e sua ordem de nascimento, com uma sobre-representacão nos primeiros e nos últimos nascidos.

\section{DISCUSSAO}

A escolha do método de Greenwood-Yule para testar efeitos de ordem de nascimento é antiga na literatura e, em estudos desta natureza, este é o método mais utilizado ${ }^{20}$. $\mathrm{O} \mathrm{x}^{2}$ modificado por Halperin é o teste mais preciso atualmente disponivel, embora a sua utilização, envolvendo a inversão da matriz quadrática obtida, seja trabalhosa e demorada, exigindo o uso de computador eletrônico em sua forma original. Com este método pudemos estudar a associação de outras patologias - esquizofrenias e neuroses - e a ordem de nascimento ${ }^{22}$. Outros métodos foram propostos ${ }^{7,18,23}$, mas não há evidência de que sejam mais precisos que $o x^{2}$ clássico. Vale notar que o método de Halperin, introduzido há 20 anos, e simplificado pelo mesmo autor há 10 anos, nunca foi utilizado na literatura, talvez pela pouca familiaridade dos médicos com modelos matemáticos que envolvam certa complexidade ${ }^{19}$. 


\begin{tabular}{|c|c|c|c|c|c|c|c|c|c|c|c|c|c|c|c|c|c|}
\hline \multirow{2}{*}{$\begin{array}{c}\text { Tamanho } \\
\text { familia }\end{array}$} & \multirow{2}{*}{ Total } & \multicolumn{16}{|c|}{ Ordem de nascimento } \\
\hline & & $1 .^{\circ}$ & $2 .^{\circ}$ & $3.0^{\circ}$ & $4 .^{\circ}$ & $5 .^{\circ}$ & $6 .^{\circ}$ & $7 .^{\circ}$ & $8 .^{\circ}$ & $9 .^{\circ}$ & $10 .^{\circ}$ & $11 .{ }^{\circ}$ & 12." & $13 .^{n}$ & $14 . "$ & $15 . "$ & $16 .^{n}$ \\
\hline 1 & 10 & 10 & & & & & & & & & & & & & & & \\
\hline 2 & 25 & 15 & 10 & & & & & & & & & & & & & & \\
\hline 3 & 31 & 10 & 6 & 15 & & & & & & & & & & & & & \\
\hline 4 & 32 & 14 & 8 & 5 & 5 & & & & & & & & & & & & \\
\hline 5 & 18 & 2 & 4 & 6 & 3 & 3 & & & & & & & & & & & \\
\hline 6 & 21 & 2 & 2 & 5 & 5 & 1 & 6 & & & & & & & & & & \\
\hline 7 & 20 & 5 & 7 & 1 & - & 1 & 3 & 3 & & & & & & & & & \\
\hline 8 & 23 & 3 & 2 & 3 & 5 & 3 & 1 & 1 & 5 & & & & & & & & \\
\hline 9 & 12 & 3 & 1 & 2 & - & - & 1 & 1 & 2 & 2 & & & & & & & \\
\hline 10 & 18 & 2 & 1 & 3 & 1 & - & 2 & 1 & 3 & 一 & 5 & & & & & & \\
\hline 11 & 9 & 1 & 1 & 1 & 1 & - & - & 1 & 1 & 1 & - & 2 & & & & & \\
\hline 12 & 7 & - & 1 & 1 & 一 & 1 & 1 & - & 1 & - & - & - & 2 & & & & \\
\hline 13 & 3 & - & - & - & - & - & 1 & - & 二 & - & $\ldots$ & - & 1 & 1 & & & \\
\hline 14 & 3 & - & 一 & - & 1 & - & 一 & $\ldots$ & - & - & - & - & - & - & 2 & & \\
\hline 15 & 3 & - & 1 & - & - & - & - & - & - & - & - & $\ldots$ & - & 1 & 1 & 一 & \\
\hline 16 & 3 & 1 & $\longrightarrow$ & - & - & - & - & 1 & - & - & - & - & - & - & - & - & 1 \\
\hline 17 & 1 & - & $\longrightarrow$ & - & - & - & - & $\ldots$ & - & - & 一 & - & $\ldots$ & - & $\ldots$ & $\rightarrow$ & 1 \\
\hline 18 & 1 & 一 & 一 & - & 一 & 一 & 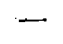 & - & - & 1 & $一$ & - & 一 & - & - & 一 & - \\
\hline Total & 238 & 68 & 44 & 40 & 21 & 9 & 14 & 9 & 12 & 4 & 5 & 2 & 3 & 2 & 3 & - & 2 \\
\hline
\end{tabular}

Tabela 2 - Valores observados nas frequências das ordens de nascimento em 2s8 epilépticos. 


\begin{tabular}{|c|c|c|c|c|c|c|c|c|c|c|c|c|c|c|c|c|c|c|c|}
\hline \multirow{2}{*}{$\begin{array}{l}\text { Lamanho } \\
\text { familia }\end{array}$} & & \multicolumn{18}{|c|}{ Ordem de nascimento } \\
\hline & & $1 .{ }^{\circ}$ & $2 .^{\circ}$ & $3 .^{\circ}$ & $4 .^{\circ}$ & $5 .^{\circ}$ & $6.0^{\circ}$ & $7 .^{\circ}$ & $8 .^{\circ}$ & $9 .^{\circ}$ & $10 .^{\circ}$ & $11 .^{\circ}$ & $12 .^{\circ}$ & $13.0^{\circ}$ & $14 .^{\circ}$ & 15.0 & $16 .^{\circ}$ & $17 .^{\circ}$ & $18 .^{\circ}$ \\
\hline 1 & 10 & 10 & & & & & & & & & & & & & & & & & \\
\hline 2 & 25 & 12,5 & 12,5 & & & & & & & & & & & & & & & & \\
\hline 3 & 31 & 9,66 & 9,66 & 9,66 & & & & & & & & & & & & & & & \\
\hline 4 & 32 & 8 & 8 & 8 & 8 & & & & & & & & & & & & & & \\
\hline 5 & 18 & 3,6 & 3,6 & 3,6 & 3,6 & 3,6 & & & & & & & & & & & & & \\
\hline 6 & 21 & 3,5 & 3,5 & $\mathbf{3 , 5}$ & 3,5 & 3,5 & 3,5 & & & & & & & & & & & & \\
\hline 7 & 20 & 2,85 & 2,85 & 2,85 & 2,85 & 2,85 & 2,85 & 2,85 & & & & & & & & & & & \\
\hline 8 & 23 & 2,8 & 2,8 & 2,8 & 2,8 & 2,8 & 2,8 & 2,8 & 2,8 & & & & & & & & & & \\
\hline 9 & 12 & $\mathbf{1 , 3 3}$ & 1,33 & 1,33 & 1,33 & 1,33 & 1,33 & 1,33 & 1,33 & 1,33 & & & & & & & & & \\
\hline 10 & 18 & 1,8 & 1,8 & 1,8 & 1,8 & 1,8 & 1,8 & 1,8 & 1,8 & 1,8 & 1,8 & & & & & & & & \\
\hline 11 & 9 & 0,81 & 0,81 & 0,81 & 0,81 & 0,81 & 0,81 & 0,81 & 0,81 & 0,81 & 0,81 & 0,81 & & & & & & & \\
\hline 12 & 7 & 0,58 & 0,58 & 0,58 & 0,58 & 0,58 & 0,58 & 0,58 & 0,58 & 0,58 & 0,58 & 0,58 & 0,58 & & & & & & \\
\hline 13 & 3 & 0,23 & 0,23 & 0,23 & 0,23 & 0,23 & 0,23 & 0,23 & 0,23 & 0,23 & 0,23 & 0,23 & 0,23 & 0,23 & & & & & \\
\hline 14 & $\mathbf{3}$ & 0,21 & 0,21 & 0,21 & 0,21 & 0,21 & 0,21 & 0,21 & 0,21 & 0,21 & 0,21 & 0,21 & 0,21 & 0,21 & 0,21 & & & & \\
\hline 15 & 3 & 0,2 & 0,2 & 0,2 & 0,2 & 0,2 & 0,2 & 0,2 & 0,2 & 0,2 & 0,2 & 0,2 & 0,2 & 0,2 & 0,2 & 0,2 & & & \\
\hline 16 & 3 & 0,23 & 0,23 & 0,23 & 0,23 & 0,23 & 0,23 & 0,23 & 0,23 & 0,23 & 0,23 & 0,23 & 0,23 & 0,23 & 0,23 & 0,23 & 0,23 & & \\
\hline 17 & 1 & 0,58 & 0,58 & 0,58 & 0,58 & 0,58 & 0,58 & 0,58 & 0,58 & 0,58 & 0,58 & 0,58 & 0,58 & 0,58 & 0,58 & 0,58 & 0,58 & 0,58 & \\
\hline 18 & 1 & 0,55 & 0,55 & 0,55 & 0,55 & 0,55 & 0,55 & 0,55 & 0,55 & 0,55 & 0,55 & 0,55 & 0,55 & 0,55 & 0,55 & 0,55 & $0, \mathbf{5 5}$ & 0,55 & 0,55 \\
\hline Total & 238 & 58,48 & 48,48 & 35,98 & 26,32 & 18,32 & 14,32 & 11,22 & 8,37 & 5,50 & 4,17 & 2,37 & 2,56 & 0,98 & 0,75 & 0,54 & 0,34 & 0,113 & 0,55 \\
\hline
\end{tabular}

Tabela 3 - Valores esperados, segundo o método de Greenwood-Yule, das freqüências das ordens de nascimento em 238 epilépticos. 


\begin{tabular}{crrr}
\hline $\begin{array}{c}\text { Ordem de } \\
\text { nascimento }\end{array}$ & \multicolumn{2}{c}{ Frequência } & $\mathrm{O} / \mathrm{E}$ \\
\cline { 2 - 3 } $1 .^{\circ}$ & 68 & 58.48 & 1.16 \\
$2 .^{\circ}$ & 44 & 48.44 & 0.93 \\
$3 .^{\circ}$ & 40 & 35.98 & 1.12 \\
$4 .^{\circ}$ & 21 & 26.32 & 0.78 \\
$5 .^{\circ}$ & 9 & 18.32 & 0.49 \\
$6.0^{\circ}$ & 14 & 14.72 & 0.95 \\
$7 .^{\circ}$ & 9 & 11.22 & 0.80 \\
$8 .^{\circ}$ & 12 & 8.37 & 1.46 \\
9 e & 21 & 16.38 & 1.28 \\
\hline Total & 238 & 238.30 & - \\
\hline
\end{tabular}

Tabela / Comparação entre as frequências observadas e esperadas das ordens de nascimento em 238 epilépticos, segundo o método de Greenwood-Yule: $x^{2}$ modificado por Halperin = 46.16; gl.l = 8; $p<<<0.01$; $x^{z}$ clássico $=6.403 ;$ g.l. $=8 ; 0.5<p<0.7$.

Em relação ao nosso achado estatístico, uma ressalva deve ser feita: a conclusão favorece a afirmativa de que há uma sobre-representação estatística de primeiros e de últimos nascidos na nossa casuística de epilépticos, e não que os epilépticos tendem a nascer nas posições extremas de suas proles. Esta última interpretação já daria uma idéia de etiopatogenia: não era este o objetivo do estudo e nem a conclusão estatística obtida permite tal inferênicia.

Não se inclui no objetivo do trabalho a explicação da natureza desta associação encontrada; conforme já notado anteriormente, esta explicação provém de vários modelos teóricos. No caso de epilepsia, talvez seja pertinente raciocinar em termos fisiológicos e genéticos. Sem nos aprofundarmos nestas discussões, que não são o objetivo do presente estudo, pode-se lembrar que é reconhecida a importância dos traumatismos de parto no desenvolvimento da epilepsia, e que os primogênitos são sempre mais susceptíveis a acidentes obstétricos. Por outro lado, os últimos nascidos acabam por enfrentar problemas em relação à motilidade uterina, com a anóxia fetal correspondente.

Deve-se acrescentar ainda que tal verificação ainda não foi feita em nosso meio e que, além disto, a epilepsia não tem sido estudada de maneira particular nos trabalhos que cuidam de ordem de nascimento. Como já foi notado, as patologias mais estudadas são as esquizofrenias e o alcoolismo. 


\section{RESUMO}

Mediante o método de Greenwood-Yule e o teste estatístico mais sensivel de que se dispõe atualmente, $o \mathrm{x}^{2}$ de Halperin, foi testada a possivel associação entre a prevalência de epilepsia e a ordem de nascimento de 238 pacientes. Estes pacientes foram obtidos em dois hospitais de São Paulo, na tentativa de se controlar sua classe social; tratava-se de epilépticos com variados transtornos psiquiátricos. Concluiu-se por uma associação estatisticamente significante entre a doença e a ordem de nascimento dos 238 pacientes, com uma sobre-representação nos primeiros e nos últimos nascidos.

\section{SUMMARY}

\section{Epilepsy and birth order}

This paper deals with birth order and epilepsy. The Greenwood-Yule's method was applied, with the most powerful statistical test available, Halperin's modified chi-square. The authors concluded that there is a consistent positive association between this illness prevalence and birth order of the patients, being first and last born over-represented, irrespective of their sibship size. The data were obtained in two psychiatric hospitals in the city of São Paulo, being social class factor qualitativelly controlled. All the 238 patients studied suffered a wide range of psychiatric symptoms.

\section{REFERENCIAS}

1. BARRY, H. \& BLANE, T. - Birth order as a method of studiyng environmental influences in alcoholism. Ann. N.Y. Acad. Sci. 197:172, 1972.

2. BASTIDE, R. - Sociologia das Doenças Mentais. Versão brasileira. Comp. Ed. Nacional, São Paulo, 1967.

3. BATESON, G.; JACKSON, D.; HALEY, J. \& WEAKLAND, J. - Toward a theory of schizophrenla. Behav. Sci. 1:251, 1956.

4. GRANVILLE-GROSSMANN, K. - Birth order and schizophrenia. Brit. J. Psychiat. 112:1119, 1966.

5. HALPERIN, M. - The use of chi-square in testing effect of birth order. Ann. Eug. 18:99, 1953.

6. HOLLINGSHEAD, A. \& REDLICH, FK. - Social Class and Mental Illness. Science Ed., New Kork, 1964.

7. KEEPING, E. S. - The problem of birth ranks. Biometrics 6:112, 1962.

8. LEIGHTON, A.; CLAUSEN, J. \& WILSON, R. - Explorations in Social Psychiatry. Basic Books ed., New York, 1957.

9. LINT, J. de - Alcoholism, birth rank and parental deprivation. Amer. J. Psychiat. 120:1062, 1964.

10. MALZBERG, B. - Is birth order related to the incidence of mental disorders. Am. J. Phys. Anthr. 24:91, 1938

11. MCMAHON, B.; PUGH, T. \& IPSEN, J. - Métodos de Epidemiologia. Verswo castelhana. Prensa Medica Mexicana, México, D.F., 1965.

12. MANTEL, N. \& HALPERIN, M. - Analysis of birth lank data. Biometrics 19:324, 1963.

13. NEWCOMB, H. B. - Environmental versus genetic interpreations of birth order effects. Eugen. Quart. 12:90, 1965. 
14. OMS, Ser. Inf. Te'n. 185 - Epidemiologia de los transtornos mentales, 1960.

15. PRICE, J. \& HARE, E. - Birth order studies: some source of bias. Brit. J. Psychiat. 115:633, 1969.

16. RANSON, J. A. - Social Psychiatry: an overview. Amer. J. Psychiat. 130: 841. 1973 .

17. ROBERTS, B. \& MYERS, J. - Family and Class Dynamics in Mental Illness. Srience Ed., New York, 1964.

18. RLSSEL-DAVIS, D. — Birth order and maternal age of homossexuals. Lancet $\mathrm{i}: 69,1962$.

19. SANDLER, P. C. - A Psiquiatria Social e a Epidemiologia dos distúrbios mentais. Rev. Med. (São Paulo) 55:329, 1971.

20: SANDLER, P. C. - Dinâmic'a na epidemiologia da esquizofrenia. Bol. Psiquiat. (Escola Paulista de Medicina) 6:59, 1973.

21. SANDLER, P. C. - Psiquiatria social: delimitação do campo e um esquema Bol. Psiquiat. (Escola Paulista de Medicina) 7:1974 (no prelo).

22. SANDLER, P. C. \& SANDLER, E. H. - Esquizofrenia, neuroses e ordem de nascimento. Anais do III Congresso Brasileiro de Psiquiatria, Rio de Janeiro, 8-14 de setembro 1974 (no prelo).

23. SLATER, E. - Birth order and maternal age of homossexuals. Lancet i:540, 1962.

24. SOLOMON, L. \& NUTTAL, R. -- Sibling order, premorbid adjustment and remission in schizophrenia. J. Nerv. Ment. Dis. 144:37, 1967.

25. SUNDARAJ, R. R. - Birth order and schizophrenia. J. Nerv. Ment. Dis. 138:87, 1964.

26. VERON, E. - Perspectivas futuras de la investigación básica sócio-cultural sobre salud mental. Acta Psiq. Psicol. Amer. Lat. 193:210, 1968.

27. ZUBIN, J. - Social Psychiatry. Grunne \& Stratton, New York, 1968.

Departamento de Medicina Preventiva - Faculdade le Medicina da Universidade de São Paulo - Av. Dr. Arnaldo 425-012.46 São Paulo, SP - Brasil. 